\title{
¿Está la morfometría de las manos relacionada con la edad de la menarquia?
}

\author{
Laura Marín M. ${ }^{1}$, Isabel Molpeceres ${ }^{1}$, Julio A. Gobernado-Tejedor ${ }^{2}$, Cristina Alvarez- \\ Colomo ${ }^{2}$, Ana García-López ${ }^{1}$, Sonia de Miguel-Manso ${ }^{2}$, José Schneider-Fontán. ${ }^{3}$ \\ ${ }^{1}$ Médico Residente de Obstetricia y Ginecología, Hospital Clínico Universitario de Valladolid. ${ }^{2}$ Licenciado Especialista en \\ Obstetricia y Ginecología, Hospital Clínico Universitario de Valladolid; Profesor Asociado de Obstetricia y Ginecología, \\ Facultad de Medicina de Valladolid. ${ }^{3}$ Catedrático de Obstetricia y Ginecología, Facultad de Medicina de Valladolid. \\ España.
}

\section{RESUMEN}

Antecedentes: La relación entre la longitud del segundo y cuarto dedo de las manos (ratio D2:D4) se ha relacionado con la exposición intrauterina a los andrógenos. Se define un patrón androgénico cuando la longitud del cuarto dedo es superior a la del segundo y el cociente es menor de 1. Este ratio se pone en relación con aspectos del desarrollo y la función reproductiva de los individuos, tales como la resistencia al ejercicio físico, el autismo, la concentración de espermatozoides o la esterilidad. Objetivo: Establecer asociación entre la ratio D2:D4 con la edad de la menarquia. Método: Estudio transversal descriptivo sobre una población no seleccionada de 188 mujeres. Se recogen las longitudes de los dedos con un calibre digital, desde una imagen en papel obtenida con un escáner. El análisis estadístico se realizó mediante el test de correlación de Pearson, considerando significativo un valor de $\mathrm{p}<0,05$. Resultados: El análisis de correlación entre los parámetros morfométricos de las manos y la edad de la menarquia, presentó relación estadísticamente significativa y directamente proporcional con la longitud del cuarto dedo en ambos manos. Conclusión: Los resultados obtenidos indican una relación entre el perfil androgénico de la morfometría de las manos y el retraso de la menarquia. Este hecho constituye un inicio en la búsqueda de relaciones entre las características morfométricas de las manos y condiciones clínicas, que podrían estar influenciadas por la exposición androgénica durante el periodo fetal.

\section{PALABRAS CLAVE: Ratio digital D2:D4, menarquia}

\section{SUMMARY}

Background: The relationship between the length of the second and fourth fingers of the hands (ratio D2:D4) has been associated with intrauterine exposure to androgens. The androgenic pattern is defined when the length of the fourth finger is higher than the second, and the ratio is lower than 1 . This ratio is set in relation to aspects of development and reproductive function of individuals, such as resistance to physical exercise, autism, sperm concentration or sterility. Aims: Find relationship between the ratio D2:D4 with age of menarche. Methods: Descriptive cross-sectional study in an unselected population of 188 women. The finger lengths with a digital caliper is collected from a paper image obtained by a scanner. Statistical analysis was perfomed using the Pearson correlation coefficient, with significant value $p<0.05$. Results: Correlation analysis between the morphometric parameters of the hands and the age of menarche, showed statistically significant and directly proportional to the length of the fourth finger on both hands relationship. Conclusion: The results indicate a relationship between the androgenic pattern morphometry hands and 
late menarche. This is a start in the search for relationships between the morphometric characteristics of the hands and clinical conditions that might be influenced by androgen exposure during fetal period.

\section{KEY WORDS: Digital ratio D2:D4, menarche}

\section{INTRODUCCIÓN}

En el ser humano, el segundo y cuarto dedo de la mano presentan un patrón simétrico respecto al tercer dedo. Sin embargo, hay una variación considerable en la ratio de la longitud del $2^{\circ}$ y $4^{\circ}$ dedo (2D:4D): algunos individuos tienen el $2^{\circ}$ dedo más largo que el $4^{\circ}(2 \mathrm{D}: 4 \mathrm{D} \geq 1)$ mientras que otros presentan el caso inverso (2D:4D $\leq 1)(1)$.

El dimorfismo sexual se manifiesta desde el periodo intrauterino, siendo la ratio 2D:4D menor en varones que en hembras. Se ha propuesto la ratio como un indicador indirecto de la actividad prenatal de andrógenos, de forma que una ratio menor de 1 , reflejaría una mayor exposición intraútero a la testosterona (2-5). Hay otras evidencias de que la ratio 2D:4D está influenciada por la exposición prenatal a los andrógenos y que esta relación es estable en el tiempo (5-7). Diversos estudios han ligado la ratio digital al desarrollo físico, orientación sexual, habilidad espacial, autismo e incluso fertilidad (3,810). La magnitud de estas asociaciones no siempre es consistente en los diferentes estudios.

El ambiente hormonal intrauterino puede influir en el momento del desarrollo puberal y consecuentemente en el inicio de los periodos menstruales $(11,12)$. Algunos estudios han investigado la posible asociación entre la edad de la menarquia y la ratio digital. Ratios bajas se han asociado a retraso de la edad de la menarquia $(13,14)$, sin embargo, esta conclusión no se ha contrastado en otros estudios (15). Un enfoque novedoso aparece a partir de los trabajos de Medland y cols (16), que encuentra una potente asociación entre una variante del gen LIN28B y la ratio 2D:4D. Esta variante se ha relacionado con la menarquia retrasada, lo que apoyaría la asociación entre la ratio digital y la edad de la menarquia (17).

El objetivo de nuestro estudio es buscar la relación entre la ratio D2:D4 con la edad de la menarquia, suponiéndole a esta un valor indirecto de la impregnación androgénica intrauterina en mujeres no seleccionadas.

\section{PACIENTES Y MÉTODO}

Se diseñó un estudio transversal descriptivo sobre una población no seleccionada de mujeres trabajadoras del Hospital Clínico Universitario de
Valladolid, que participaron tras la información y consentimiento verbal. Se realizó la captación de la imagen de la cara palmar de las manos con un escáner, con los dedos separados (Figura 1 y 2). Se obtuvo, por un mismo observador, la longitud del segundo y cuarto dedo de cada mano, sobre papel impreso utilizando un calibre digital con una precisión de 0,01 $\mathrm{mm}$. La medición de cada dedo se realizó desde el pliegue basal hasta el límite cutáneo de la tercera falange, siguiendo la metodología propuesta por Manning y cols (3) (Figura 3). Se recogieron los datos de la edad actual de la participante y la edad de la menarquia. El análisis estadístico se realizó mediante el test de correlación de Pearson, se consideró un valor de $p<0,05$ como significativo. Se utilizó el paquete IBM SPSS Statistics 22. El estudio fue autorizado por la Comisión de Ética e Investigación del Hospital Clínico Universitario de Valladolid.

\section{RESULTADOS}

Se ofreció participar en el estudio a 200 mujeres de las cuales 188 accedieron. La mediana de edad actual de las participantes fue 38 años (Q1Q3: 33-52 años) con un mínimo de 20 y un máximo de 63 años. La edad de la menarquia presentó una mediana de 12 años (Q1-Q3: 11-14 años) con un mínimo de 9 y un máximo de 16 años.

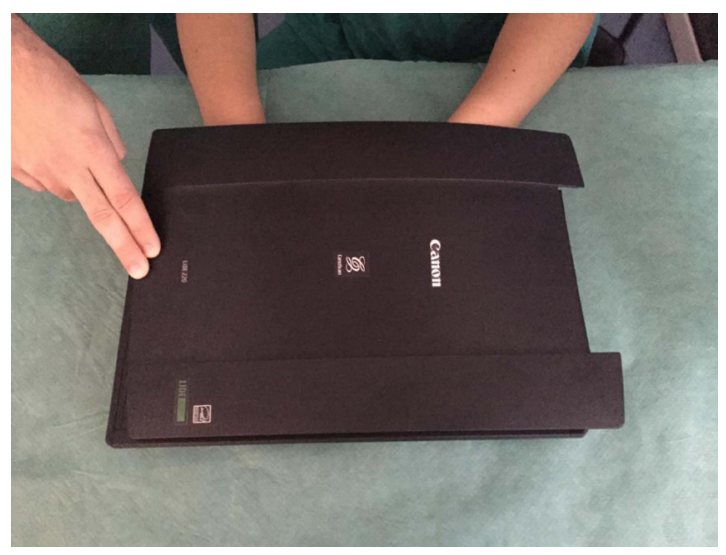

Figura 1. Metodología para capturar la imagen palmar de las manos, mediante un escáner. 


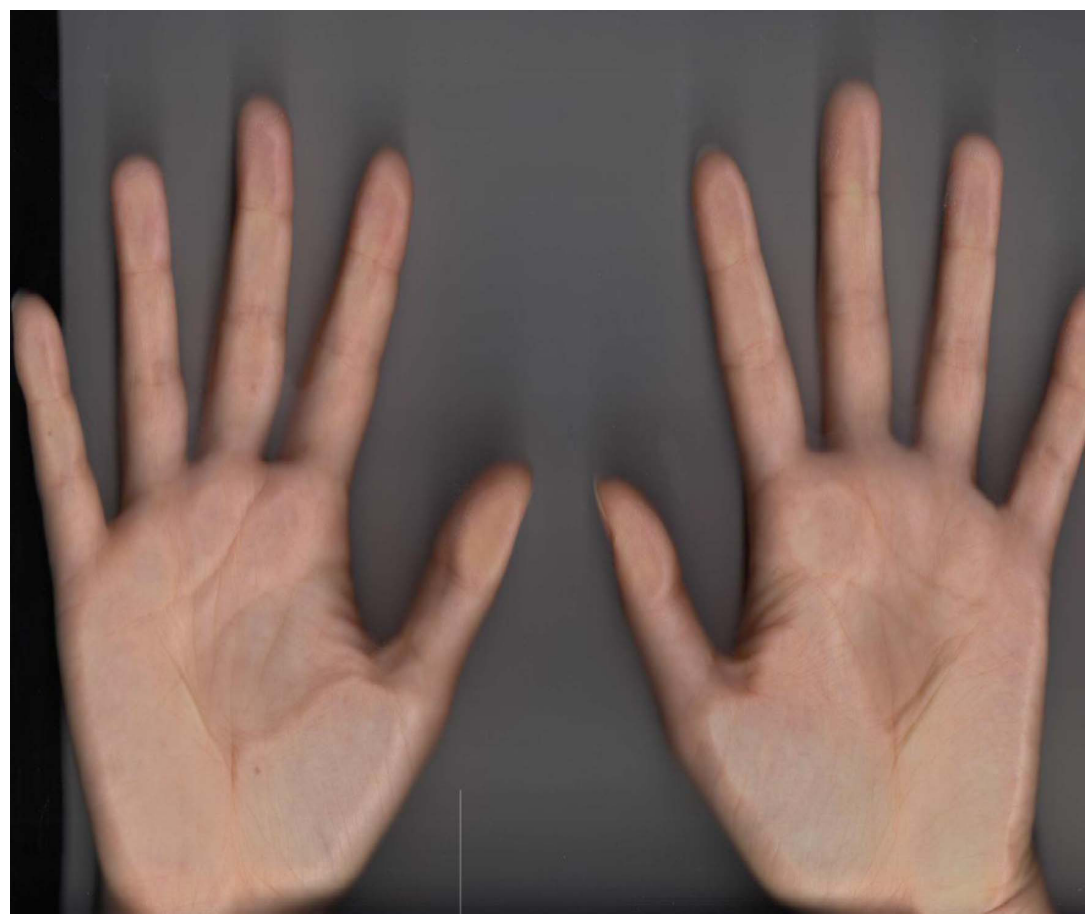

Figura 2. Imagen obtenida mediante el escáner de la cara palmar de ambas manos.

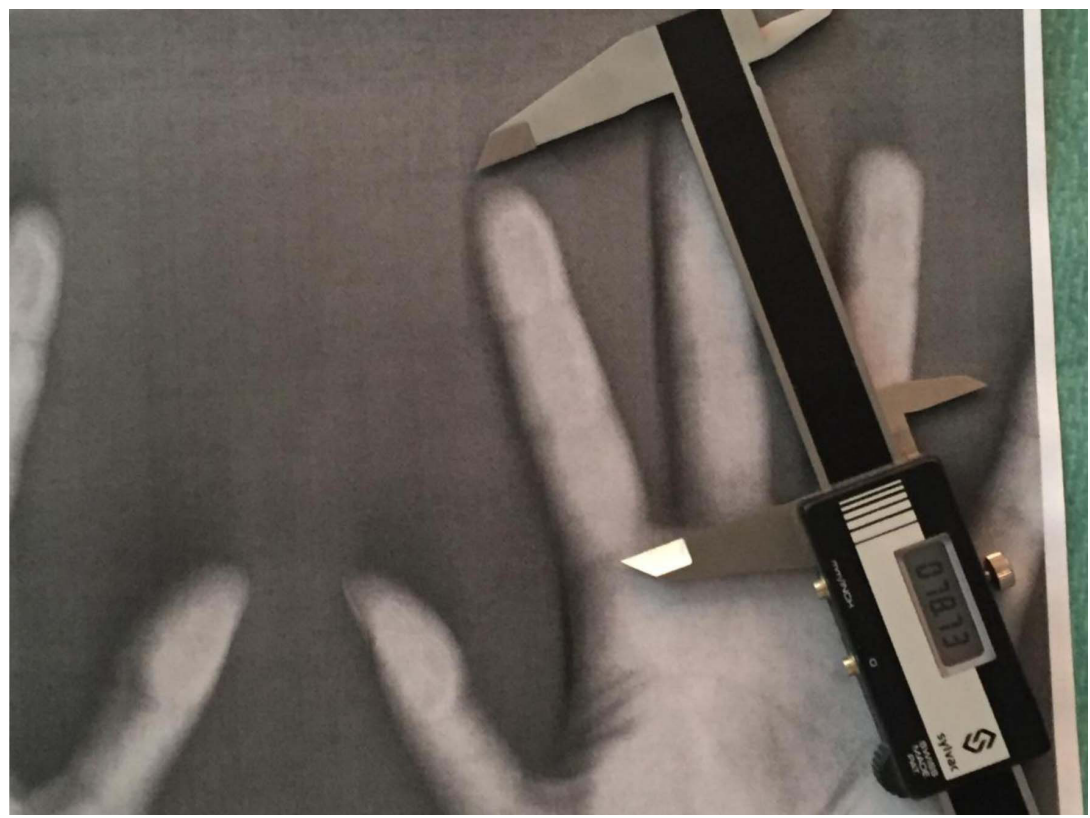

Figura 3. Metodología de la medida de la longitud digital, con calibre digital, desde una copia en papel de la imagen obtenida mediante el escáner. 
El análisis de correlación entre los parámetros morfométricos de las manos y la edad de la menarquia, presentó una relación estadísticamente significativa y directamente proporcional con la longitud del cuarto dedo en ambas manos (Tabla I). No resultó significativa la relación con la longitud del segundo dedo ni con la ratio D2:D4. En las Figuras 4 y 5 se muestra la gráfica de puntos con la línea de regresión.

\section{DISCUSIÓN}

Desde su descubrimiento en 1978, los genes Hox han cautivado a un grupo diverso de investigadores, desde biólogos del desarrollo a clínicos puros. Se trata de un grupo de genes evolutivamente conservados. Codifican una familia de factores de transcripción que regulan los primeros procesos morfogenéticos y de desarrollo, y continuarán siendo expresados en la edad adulta. En los vertebrados hay un total de 39 genes Hox organizados en 4 grupos distintos. En los seres humanos, los 4 grupos contienen entre 9 y 11 genes y se localizan en 4 cromosomas diferentes. Estas agrupaciones, denominadas HOXA, HOXB, HOXC y HOXD, se encuentran en los cromosomas $7 p 14,17 q 21,12 q 13$ y 2q31, respectivamente (18).

Tabla I

COEFICIENTES DE CORRELACIÓN DE LA MENARQUIA Y LOS PARÁMETROS MORFOMÉTRICOS DE LAS MANOS

\begin{tabular}{|c|c|c|c|c|c|c|c|}
\hline$n$ & $\begin{array}{l}\mathrm{D} 2 \mathrm{R} \\
188\end{array}$ & $\begin{array}{l}\mathrm{D} 4 \mathrm{R} \\
188\end{array}$ & $\begin{array}{l}\text { D2L } \\
188\end{array}$ & $\begin{array}{l}\mathrm{D} 4 \mathrm{~L} \\
188\end{array}$ & $\begin{array}{c}\text { D2D4R } \\
188\end{array}$ & $\begin{array}{c}\text { D2D4R } \\
188\end{array}$ & $\begin{array}{c}\mathrm{RL} \\
188\end{array}$ \\
\hline $\begin{array}{l}\text { Correlación de } \\
\text { Pearson }\end{array}$ & 0,118 & 0,158 & 0,140 & 0,144 & $-0,059$ & $-0,021$ & $-0,040$ \\
\hline $\begin{array}{l}\text { Valor } p \\
\text { (two tails) }\end{array}$ & 0,107 & $0,031^{*}$ & 0,056 & $0,048^{*}$ & 0,422 & 0,776 & 0,585 \\
\hline
\end{tabular}

$\mathrm{D}=$ dedos. D2D4R=Ratio D2:D4 mano derecha. D2D4L=Ratio D2:D4 mano izquierda. RL=Ratio D2D4R:D2D4L. * $\mathrm{p}<0,05$.

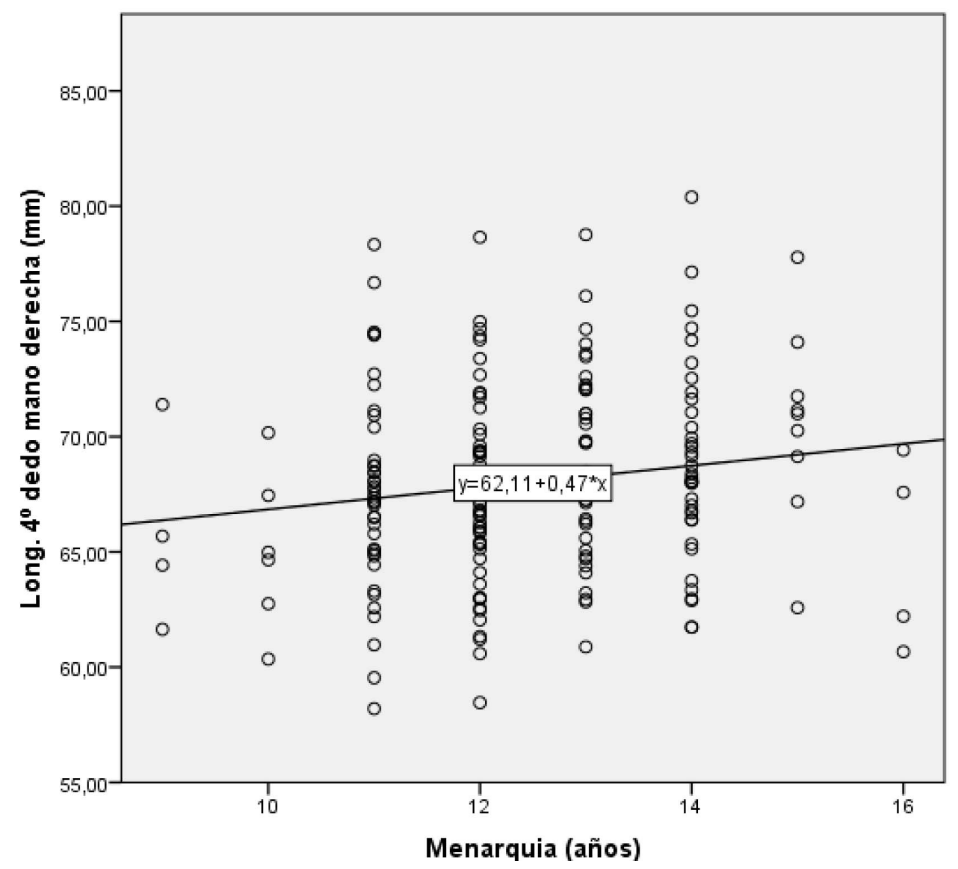

Figura 4. Relación edad de la menarquia-longitud de 4ํ dedo mano derecha. 


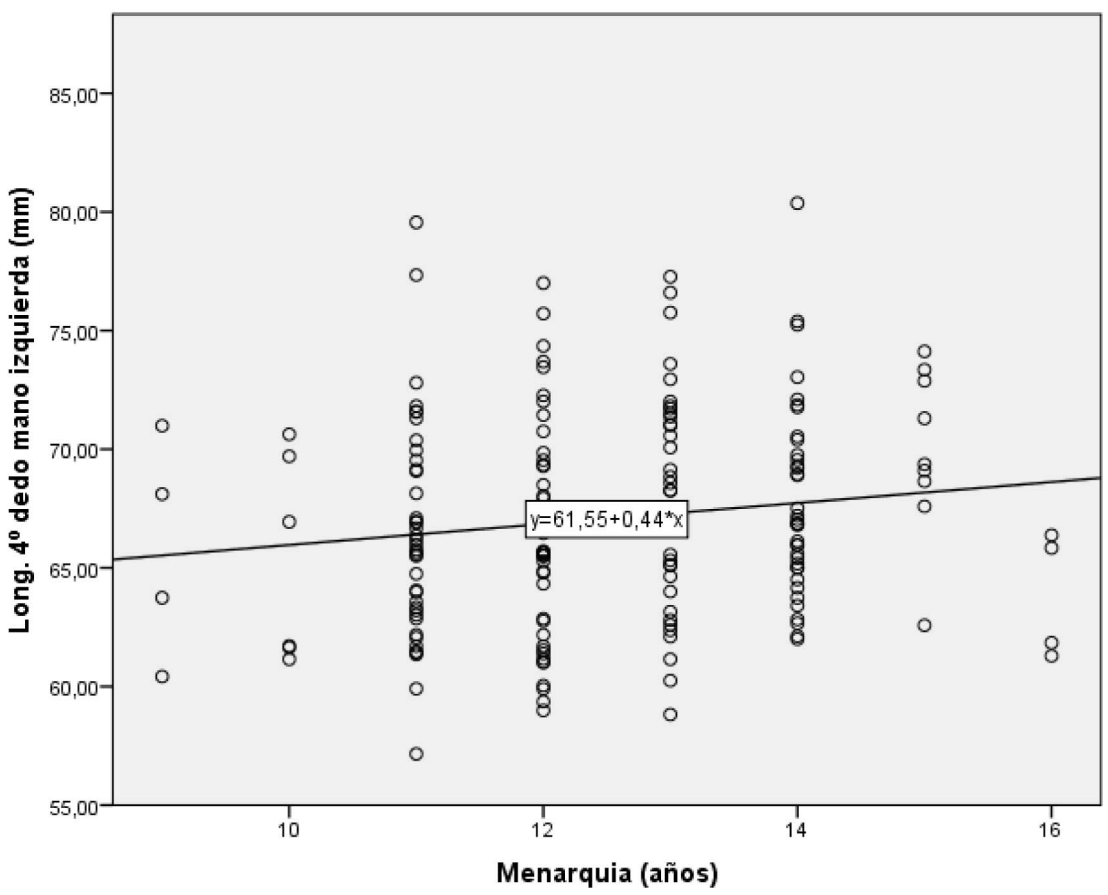

Figura 5. Relación edad de la menarquia-longitud de $4^{\circ}$ dedo mano izquierda.

El gen Hox es esencial para el desarrollo de las extremidades y el aparato genital en los vertebrados (19). Participa en el crecimiento y diferenciación de los dedos y del tubérculo genital que dará lugar al aparato urogenital (20). Se sabe que mutaciones del gen HOXA13 causan defectos congénitos a nivel de los dedos y los genitales (Síndrome mano-pie-genital de herencia autosómica dominante; OMIN \#140000).

Matchock (13), en 2008, demostró por primera vez una asociación entre ratios D2:D4 menores de 1 (predominio androgénico), con edades más avanzadas de la primera menstruación. Este autor sólo encontró asociación para los valores morfométricos de la mano derecha. Tres años después Manning y Fink (14) corroboran la relación de la edad de la menarquia con los valores morfométricos de ambas manos. Las mediciones, en este caso, estaban realizadas por las propias participantes en el estudio, un total de 70.658 mujeres caucásicas, que aportaban además de la longitud de los dedos, la edad de su menarquia a través de internet de forma voluntaria. Sin embargo, Oberg y Villamor (21), en un estudio prospectivo de 299 adolescentes colombianas, midió la longitud del segundo y cuarto dedo periódicamente hasta la edad de la menarquia, y solo pudo confirmar la relación con la ratio en la mano derecha.

Nuestros resultados no permiten confirmar una relación estadísticamente significativa entre la edad de la menarquia y la ratio D2:D4. Sin embargo, sí encontramos una relación directamente proporcional entre la edad de aparición de la primera menstruación y la longitud del cuarto dedo, de forma que longitudes mayores corresponderían a mujeres con menarquia más tardía. Esta relación se ha puesto de manifiesto para ambas manos entre las participantes en nuestro estudio. Estos resultados estarían en consonancia con los trabajos de Matchock (13), Manning y Fink (14) y Oberg y Villamor (21) e indicarían una relación entre el perfil androgénico de la morfometría de las manos y el retraso de la menarquia. Tanto la ratio D2:D4 menor de uno, como la mayor longitud del cuarto dedo de forma aislada, estarían relacionadas con el patrón androgénico.

La diferente metodología en la obtención de los datos y el análisis de los mismos, podría estar relacionada con la ausencia de concordancia en los resultados de los estudios publicados. Únicamente en el estudio de Oberg y Villamor (21), el equipo investigador realizó la medición de la longitud digital 
en todos los casos. Estos autores, realizan además un análisis incluyendo factores de confusión como la altura y el índice de masa corporal.

La fortaleza de nuestro trabajo radica en que la medición de la longitud de los dedos se ha realizado en todos los casos por el mismo investigador, utilizando un calibre digital con una precisión de una micra. Este hecho limita el error en la medición, al eliminar la variabilidad interobservador. Por el contrario, los autores reconocen como limitación la falta de análisis de las características antropométricas de las participantes. Aunque el propio diseño del estudio con inclusión de mujeres adultas e incluso postmenopaúsicas, hacen poco probable que las medidas actuales de las participantes sean equiparables a las del momento de su menarquia.

\section{CONCLUSIÓN}

Existe una relación significativa y directamente proporcional entre la edad de aparición de la primera menstruación y la longitud del cuarto dedo en ambos manos, de forma que longitudes mayores corresponderían a mujeres con menarquia más tardía, en la muestra estudiada de mujeres no seleccionadas. Este trabajo es el inicio de una línea de investigación muy interesante, en la que el equipo investigador pretende buscar la posible relación de las características morfométricas de las manos de las mujeres con condiciones clínicas importantes, que podrían estar influenciadas por la exposición a andrógenos en el periodo fetal.

\section{REFERENCIAS}

1. Malas MA, Dogan S, Evcil EH, Desdicioglu K. Fetal development of the hand, digits and digit ratio (2D:4D). Early Hum Dev. 2006;82(7):469-75.

2. Manning J, Kilduff $L$, Cook C, Crewther B, Fink B. Digit Ratio (2D:4D): A biomarker for prenatal sex steroids and adult sex steroids in challenge situations. Front Endocrinol (Lausanne). 2014;5:9.

3. Manning JT, Scutt D, Wilson J, Lewis-Jones DI. The ratio of 2 nd to 4 th digit length: a predictor of sperm numbers and concentrations of testosterone, luteinizing hormone and oestrogen. Hum Reprod. 1998;13(11):3000-4.

4. Honekopp J, Watson S. Meta-analysis of digit ratio 2D:4D shows greater sex difference in the right hand. Am J Hum Biol. 2010;22(5):619-30.

5. McIntyre MH, Cohn BA, Ellison PT. Sex dimorphism in digital formulae of children. Am J Phys Anthropol. 2006;129(1):143-50.
6. Breedlove SM. Minireview: Organizational hypothesis: instances of the fingerpost. Endocrinology. 2010;151(9):4116-22.

7. Trivers R, Manning J, Jacobson A. A longitudinal study of digit ratio (2D:4D) and other finger ratios in Jamaican children. Horm Behav. 2006;49(2):150-6.

8. Manning JT, Baron-Cohen S, Wheelwright S, Sanders $\mathrm{G}$. The 2 nd to 4 th digit ratio and autism. Dev Med Child Neurol. 2001;43(3):160-4.

9. Manning JT, Churchill AJ, Peters M. The effects of sex, ethnicity, and sexual orientation on self-measured digit ratio (2D:4D). Arch Sex Behav. 2007;36(2):223-33.

10. Puts DA, McDaniel MA, Jordan CL, Breedlove SM. Spatial ability and prenatal androgens: meta-analyses of congenital adrenal hyperplasia and digit ratio (2D:4D) studies. Arch Sex Behav. 2008;37(1):100-11.

11. D'Aloisio AA, DeRoo LA, Baird DD, Weinberg CR, Sandler DP. Prenatal and infant exposures and age at menarche. Epidemiology. 2013;24(2):277-84.

12. Epplein M, Novotny R, Daida Y, Vijayadeva V, Onaka AT, Le Marchand L. Association of maternal and intrauterine characteristics with age at menarche in a multiethnic population in Hawaii. Cancer Causes Control. 2010;21(2):259-68.

13. Matchock RL. Low digit ratio (2D:4D) is associated with delayed menarche. Am J Hum Biol. 2008;20(4):487-9.

14. Manning JT, Fink B. Is low digit ratio linked with late menarche? Evidence from the BBC internet study. Am J Hum Biol. 2011;23(4):527-33.

15. Helle S. Does second-to-fourth digit length ratio (2D:4D) predict age at menarche in women? Am J Human Biol. 2010;22(3):418-20.

16. Medland SE, Zayats T, Glaser B, Nyholt DR, Gordon $\mathrm{SD}$, Wright MJ, et al. A variant in LIN28B is associated with 2D:4D finger-length ratio, a putative retrospective biomarker of prenatal testosterone exposure. Am J Hum Genet. 2010;86(4):519-25.

17. He C, Kraft P, Chen C, Buring JE, Pare G, Hankinson $\mathrm{SE}$, et al. Genome-wide association studies identify loci associated with age at menarche and age at natural menopause. Nat Genet. 2009;41(6):724-8.

18. Quinonez SC, Innis JW. Human HOX gene disorders. Mol Genet Metab. 2014;111(1):4-15.

19. Herault Y, Fraudeau N, Zakany J, Duboule D. Ulnaless (UI), a regulatory mutation inducing both loss-offunction and gain-of-function of posterior Hoxd genes. Development. 1997;124(18):3493-500.

20. Kondo T, Zakany J, Innis JW, Duboule D. Of fingers, toes and penises. Nature. 1997;390(6655):29.

21. Oberg AS, Villamor E. Low digit ratio predicts early age at menarche in Colombian schoolgirls. Paediatr Perinat Epidemiol. 2012;26(5):448-55. 